EESTI NSV TEADUSTE AKADEEMIA TOIMETISED. XV KÖIDE FOOSIKA-MATEMAATIKA- JA TEHNIKATEADUSTE SEERIA. 1966, NR. 1

ИЗВЕСТИЯ АКАДЕМИИ НАУК ЭСТОНСКОИ ССР. ТОМ ХV СЕРИЯ ФИЗИКО-МАТЕМАТИЧЕСКИХ И ТЕХНИЧЕСКИХ НАУК. 1966, № 1

M. РУБЕЛЬ

\title{
СТАТИСТИЧЕСКАЯ ХАРАКТЕРИСТИКА ВЫПУКЛОСТИ СТВОРОК БРАХИОПОД С ПЛОСКОЙ ПОВЕРХНОСТЬЮ СМЫКАНИЯ
}

Как известно [4], рисунок внешней поверхности выпуклой створки брахиопод, расположенный на плоскости симметрии и образующийся в процессе их рогта, в определенной степени соответствует росту логарифмической спирали раковины. На этом основании для некоторых типов раковин можно выработать простые приемы и объективные критерии для характеристики выпуклости створок брахиопод, что и является задачей настоящей статьи.

Рассматриваем только брахиоподы с плоской поверхностью смыкания *. Называем последнюю плоскостью смыкания. Определяем поверхность створки только как ее внешнюю поверхность без палинтропа. Қак уже отмечалось, поверхность створки образует на плоскости симметрии рисунок, соответствующий логарифмической спирали до образования так называемой вертикальной зоны роста [ $\left.{ }^{4}\right]$.

Логарифмическая спираль имеет в полярных координатах следующее уравнение [1]:

$$
\varrho=e^{k \varphi},
$$

где $\varrho$ и $\varphi$ - координаты точек спирали и $e$ - основание натуральных логарифмов;

по определению спирали

$$
k=\operatorname{ctg} \alpha,
$$

где $\alpha$ - постоянный угол для данной спирали, под которым она пересекает все лучи, выходящие из начала координат.

Предел длины дуги от начала $\left(l_{0}\right)$ выражается формулой

$$
i_{0}=\frac{\sqrt{1+k^{2}}}{k} \mathrm{Q} \text {. }
$$

Отсюда видно, что по значениям $l_{0}$ и $\varrho$ можно найти величину $k$, которая определяет выпуклость данной спирали. Эту же величинку $k$ принимаем и за меру выпуклости створки **.

* Поверхность смыкания может быть у замковых брахиопод поверхностью 1-го порядка (= плоскостью), 2-го порядка и т. д.

** Выпуклость створок определена как «расстояние между разделяющей плоскостью (= плоскостью смыкания. - M.P.) и параллельной к ней плоскостью касательной к поверхности створки ...» ([2], стр. 121). Это не позволяет определить форму раковины однозначно. Поэтому в описаниях брахиопод чаще пользуются различными отношениями выпуклости к каким-нибудь другим размерным признакам створки. Последний способ выражения выпуклости по существу принят и здесь. 
Величинам $l_{0}$ и $\varrho$ на раковине брахиоподы соответствуют с определенным недостатком или излишком (см. ниже) пары размеров $x_{3}$ и $x_{1}$, $x_{1}$ и $x_{2}$ (рис. 1 и 2 ).

Для дальнейшего определим эти признаки на плоскости симметрии раковины. При этом целесообразно рассматривать отдельно створки с палинтропом (ареей) и без него (на рис. 1 и 2 соответственно брюшная $(V)$ и спинная $(D)$ створки).

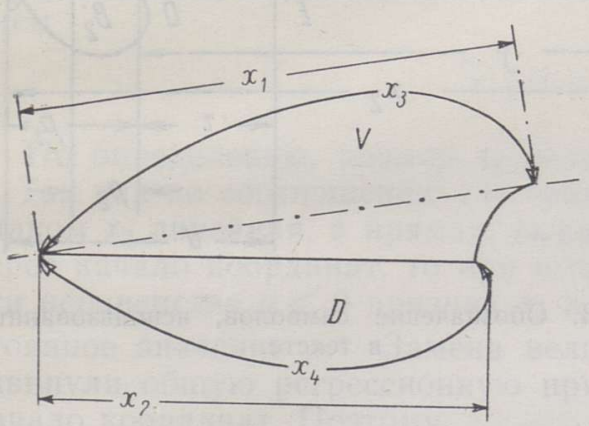

Рис. 1. Размеры брюшной $(V)$ и спинной $(D)$ створок на плоскости симметрии. Линии $x_{3}$ и $x_{4}$ вычерчены по значению $k=1,5$.

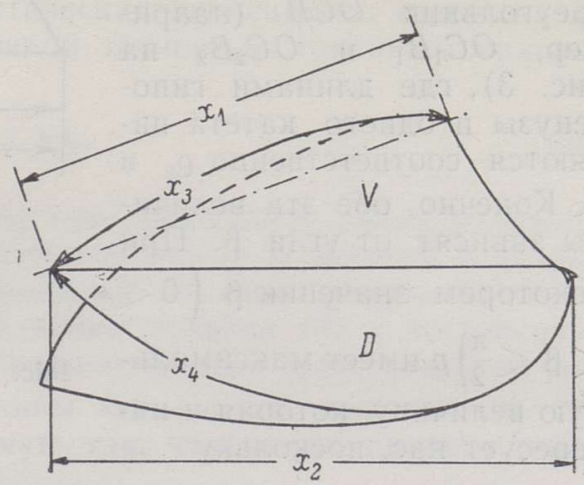

Рис. 2. Размеры $x_{i}(i=1,2,3,4)$ раковины с синусом на брюшной $(V)$ и седлом на спинной $(D)$ створках. Линия $x_{3}$ вычерчена по значению $k=2,5$ и линия $x_{4}-$ по значению $k=1,0$.

Определения следующие: при створках с палинтропом $x_{1}$ - расстояние между наиболее удаленными точками поверхности створки, проектируемыми на линию, проходящую через начало роста и точку пересекания передней части створки с плоскостью смыкания; $x_{3}-$ длина поверхности створки, т. е. длина дуги от начала роста до плоскости смыкания в передней части створки. При створках без палинтропа $x_{2}-$ расстояние между наиболее удаленными точками поверхности створки, проектируемыми на плоскость смыкания, а $x_{4}$ определяется так же, как и $x_{3}$.

$\mathrm{y}$ раковин с синусом и седлом точно измерить определенные выше признаки на плоскости симметрии неудобно. В таком случае основой измерения является боковой вид раковины без учета синуса и седла (см. рис. 2). Әто значит, что фактически мы измеряем проекции йтересующих нас признаков, находящихся на плоскости данного разреза под некоторым углом.

Исходя из вышеопределенных размеров, измеренных на серии экземпляров (выборке) какой-нибудь популяции, найдем статистическую характеристику выпуклости поверхности створок этой выборки. Сперва: найдем такую характеристику для створок без палинтропа.

Из рис. 3 видно, что признак $x_{2}$ при любой стадии роста больше соответствуюшей величины @. Наоборот, признак $x_{4}$ меньше соответствующей величины $l_{0}$, так как начало роста иногда не видно (например у спинной створки целой раковины) или просто не поддается измерению с нужной точностью (раковина разрушена последующими стадиями роста, например, у брюшной створки). Следует отметить, что начало роста не совпадает точно с началом логарифмической спирали. Поэтому необходимо более подробно рассмотреть соотношения между приведенными размерами $x_{i}(i=2,4)$ и аналогичными величинами логарифмической: спирали (е и $\left.l_{0}\right)$. 
Докажем, что имеет место соотношение

$$
\frac{l_{0}}{x_{2}}=C=\text { const. }
$$

Рассмотрим при случайно выбранном значении движущийся прямоугольный треугольник $O C B$ (напримep, $O C_{1} B_{1}$ и $O C_{2} B_{2}$ на рис. 3), где длинами гипотенузы и одного катета являются соответственно $\varrho_{3}$ и p. Конечно, обе эти величины зависят от угла $\beta$. При некотором значении $\beta(0<$ $\left.<\beta<\frac{\pi}{2}\right) p$ имеет максимальную величину, которая и интересует нас, поскольку

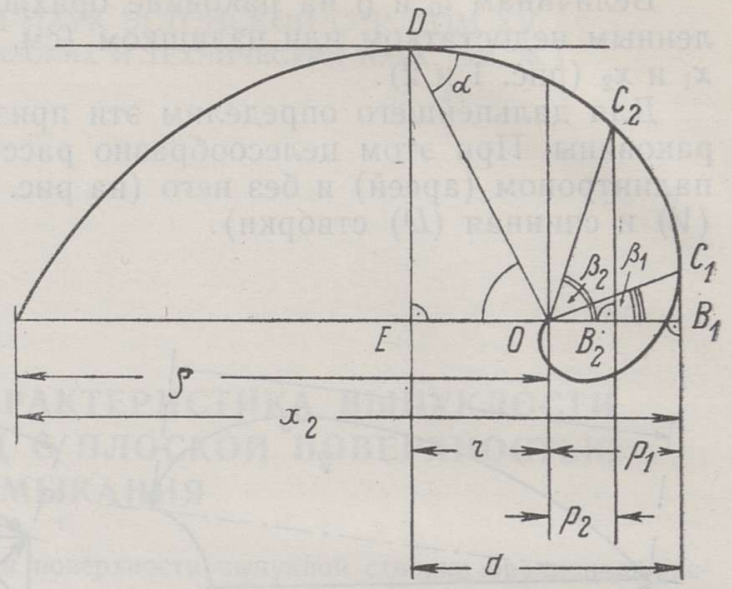

Рис. 3. Обозначение символов, использованных в тексте.

$$
x_{2}=\varrho+p_{\max }
$$

Из треугольника $O C B$ видно, что $p=\varrho_{\beta} \cos \beta$. Так как $\varrho_{\beta}=e^{k(\varphi-\pi+\beta)}$, то можно написать

$$
p=e^{k(\varphi-\pi+\beta)} \cos \beta .
$$

Для определения максимального значения $p$ дифференцируем последнее выражение по $\beta$ и приравниваем его к нулю, т. е.

$$
p^{\prime}=e^{k(\varphi-\tau+\beta)}(k \cos \beta-\sin \beta)=0 .
$$

Отсюда видно, что $k \cos \beta-\sin \beta=0$. Следовательно, значение $\beta$ можно определить из тригонометрического уравнения

$$
\beta=\operatorname{arctg} k .
$$

Принимая во внимание область изменения величины $\beta$, получим

$$
\cos \beta=\frac{1}{\sqrt{1+k^{2}}} \text {. }
$$

Подставляя найденные соотношения в формулу (6), получим

$$
p_{\max }=\frac{e^{k \varphi} \cdot e^{k \operatorname{arctg} k}}{e^{k \pi} \sqrt{1+k^{2}}}=\frac{e^{k \operatorname{arctg} k}}{e^{k \pi} \sqrt{1+k^{2}}} \varrho .
$$

Тогда из уравнения (5) найдем, что

$$
\varrho=\frac{e^{k \pi} \sqrt{1+k^{2}}}{e^{k \pi} \sqrt{1+k^{2}}+e^{k \operatorname{arctg} k}} x_{2},
$$

T. e.

$$
\frac{x_{2}}{\varrho}=\frac{e^{k \pi} \sqrt{1+k^{2}}}{e^{k \pi} \sqrt{1+k^{2}}+e^{k \operatorname{arctg} k}}=\text { const. }
$$




\section{Отсюда при помощи соотношения (3) получим}

$$
\frac{l_{0}}{x_{2}}=\frac{\left(1+k^{2}\right) e^{k \pi}}{k\left(e^{k \pi} \sqrt{1+k^{2}}+e^{k \operatorname{arctg} k}\right)}=C=\text { const, }
$$

что и требовалось доказать.

Из практики явствует, что статистическая зависимость признака $x_{4}$ от признака $x_{2}$ передается с достаточной точностью линейным уравнением

$$
\bar{x}_{4.2}=a+b x_{2} \text {. }
$$

По определению, размер $x_{4}$ только приблизительно равен величине $l_{0}$. Так как по соотношению (4) зависимость между величиной $l_{0}$ и признаком $x_{2}$ линейная, а прямая, выражающая эту зависимость, проходит через начало координат, то при выполнении условия (8) и достоверности неравенства $a<0$ признак $x_{4}$ в среднем меньше величины $l_{0}$ на постоянное значение $a$ * Замена величины $\bar{x}_{4.2}-a=l_{0}$ значит, что мы сдвинули общую регрессионную прямую так, чтобы она прошла через начало координат. Поэтому

$$
b=C=\frac{\left(1+k^{2}\right) e^{k \pi}}{k\left(e^{k \pi} \sqrt{1+k^{2}}+e^{k \operatorname{arctg} k}\right)} .
$$

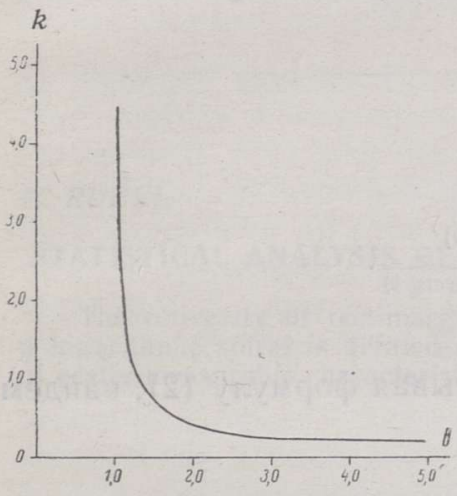

Рис. 4. График функции $k=f(b)$.

Но нас интересует величина $k$ как функция от $b$. Поскольку из уравнения (9) трудно найти нужную нам аналитическую функцию, то используем здесь для представленных целей график (рис. 4).

Значение $b$ найдено из уравнения регрессии (8). Следовательно, из графика получим среднее значение $\bar{k}$ выпуклости створок изученной выборки. Дисперсию $k$ целесообразно определить по отдельным значениям выпуклости каждого экземпляра $k_{(i)}$, оцененным через $b_{(i)}(i-$ порядковый номер каждого экземпляра) по формуле

$$
b_{(i)}=\frac{x_{4}+a}{x_{2}}(\text { cp. [3]) }
$$

По величинам $b_{(i)}$, определяющим значения $k_{(i)}$, можно вычислить дисперсию **

$$
s_{k}^{2}=\frac{\sum_{i=1}^{n}\left(k_{(i)}-\bar{k}\right)^{2}}{n-2} .
$$

* Из возможных причин возникновения неравенства $l_{0}>x_{4}$ наибольшее значение имеет несовпадение начала роста $с$ асимптотической точкой полюса $O$ спирали. Это обстоятельство дает и рациональное объяснение постоянству расхождения значений $l_{0}$ и $x_{4}$. Следует также отметить, что признак $x_{4}$ измеряется на отдельных спинных створках точнее, чем на раковинах.

** Отметим, что вследствие уравнения (9) случайные величины $b_{(i)}$ и $k_{(i)}$ не могут быть распределены одновременно по одному и тому же закону распределения. 
Изложенное, иллюстрированное на примере створок без палинтропа, действительно и для створок с палинтропом, если $x_{1}$ и $x_{3}$ измерены по приведенным определениям (см. стр. 144-145).

Непосредственным следствием роста створки по некоторой логарифмической спирали является постоянство расположения наивысшей точки створки без палинтропа относительно ее длины. Это отношение также можно выразить через величину $k$.

Для этого докажем, что имеет место соотношение

$$
\frac{d}{x_{2}}=\frac{r+p}{Q+p}=\text { const. }
$$

Из прямоугольного треугольника $O D E$ (см. рис. 3) видно, что

$$
r=Q_{\alpha} \cos \alpha \text {. }
$$

Так как $Q \alpha=e^{k(\varphi-\alpha)}, \quad$ то

$$
r=e^{k(\varphi-\alpha)} \cos \alpha=\frac{e^{k \varphi} \operatorname{ctg} \alpha}{e^{k \alpha} \sqrt{1+\operatorname{ctg}^{2} \alpha}}
$$

Учитывая формулу (3), получим

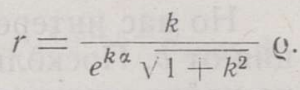

Тепгерь при помощи формулы (7) найдем

$$
\frac{d}{x_{2}}=\frac{k e^{k \pi}+e^{k(\alpha+\operatorname{arctg} k)}}{e^{k(\alpha+\pi)} \sqrt{1+k^{2}}+e^{k(\alpha+\operatorname{arctg} k)}} .
$$

Поскольку $\operatorname{arctg} k+\operatorname{arcctg} k=\frac{\pi}{2}$, то, учитывая формулу (2), найдем

$$
\frac{d}{x_{2}}=\frac{k e^{\frac{k \pi}{2}}+1}{e^{k(\pi-\operatorname{arctg} k)} \sqrt{1+k^{2}}+1} .
$$

Отсюда

$$
\frac{d}{x_{2}}=\text { const }
$$

что и требовалось доказать.

При наличии палинтропа расположение наивысшей точки створки зависит кроме величины $k$ еще от высоты и наклона палинтропа. Поэтому надо отметить, что в этом случае расположение наивысшей точки створки изменяется в процессе роста последней и, следовательно, эту точку нельзя использовать как диагностический признак.

Из всего изложенного следует вывод, что при соответствии роста створок логарифмической спирали регрессия рассмотренных признажов должна иметь вид уравнения (8). 


\section{ЛИТ Е РАТ У РА}

1. Бронштейн. И. Н. и Семендяев К. А., Справочник по математике для инженеров и учащихся втузов, М., 1964.

2. Лихарев Б. К., Основы палеонтологии, том: Мшанки, брахиоподы, Общая часть, М., 1960.

3. Рубель М. П., О применении секвенциальных критериев при определении искюпаемых брахиопод, Изв. АН ЭССР. Сер. физ.-матем. и техн. наук, 13, № 3, 1963.

4. Rudwick, M. J. S., The growth and form of brachiopod shells, Geol. Mag., Vol. XCVI, No. 1, 1959.

Ннститут геологии

Академии наук Эстонской ССР
Поступила в редакцию

17/IV 1965

M. RUBEL

\section{TASAPINNALISE UHTEPINNAGA BRAHHIOPOODIDE KUMERUSE STATISTILINE ISELOOMUSTUS}

Artiklis näidatakse, et tasapinnalise ühtepinnaga brahhiopoodide karbipoolmike kumerust, mis moodustub karbi kasvamisel logaritmilise spiraali järgi, on võimalik statistiliselt iseloomustada kahe kergesti mõõdetava tunnuste paari ( $x_{3}$ ja $x_{1}, x_{4}$ ja $x_{2}$, vt. joon. 1 ja 2 ) regressioonikoefitsientide järgi.

\section{RUBEL}

\section{STATISTICAL ANALYSIS OF CONVEXITY OF RECTIMARGINATE BRACHIOPODS}

The convexity of rectimarginate brachiopods in case of the growth of the valve by a logarithmic spiral is defined statistically by the coefficients of regression of two pairs of easily measurable characteristics: $x_{3}$ and $x_{1}, x_{4}$ and $x_{2}$ (see figs 1 and 2). 